\title{
The endosomal-lysosomal system: from acidification and cargo sorting to neurodegeneration
}

\author{
Yong-Bo Hu${ }^{1 \dagger}$, Eric B Dammer ${ }^{2 \dagger}$, Ru-Jing Ren ${ }^{1 *}$ and Gang Wang ${ }^{1 *}$
}

\begin{abstract}
The endosomal-lysosomal system is made up of a set of intracellular membranous compartments that dynamically interconvert, which is comprised of early endosomes, recycling endosomes, late endosomes, and the lysosome. In addition, autophagosomes execute autophagy, which delivers intracellular contents to the lysosome. Maturation of endosomes and/or autophagosomes into a lysosome creates an unique acidic environment within the cell for proteolysis and recycling of unneeded cellular components into usable amino acids and other biomolecular building blocks. In the endocytic pathway, gradual maturation of endosomes into a lysosome and acidification of the late endosome are accompanied by vesicle trafficking, protein sorting and targeted degradation of some sorted cargo. Two opposing sorting systems are operating in these processes: the endosomal sorting complex required for transport (ESCRT) supports targeted degradation and the retromer supports retrograde retrieval of certain cargo. The endosomal-lysosomal system is emerging as a central player in a host of neurodegenerative diseases, demonstrating potential roles which are likely to be revealed in pathogenesis and for viable therapeutic strategies. Here we focus on the physiological process of endosomal-lysosomal maturation, acidification and sorting systems along the endocytic pathway, and further discuss relationships between abnormalities in the endosomal-lysosomal system and neurodegenerative diseases, especially Alzheimer's disease (AD).
\end{abstract}

Keywords: The endosomal-lysosomal system, Neurodegeneration, Acidification, ESCRT, Retromer, Alzheimer's disease

The endosomal-lysosomal system is a series of organelles in the endocytic pathway where various cargo molecules required for normal cellular function are internalized, recycled and modulated. Recently, mounting evidence has suggested that abnormalities in both endosomes and lysosomes, or dysregulation in their trafficking, play an important role directly in a surprising host of neurological dysfunctions, represented by AD, Parkinson's disease (PD), and Lewy body dementia (LBD) [1-3]. Thus, the endosomal-lysosomal system is emerging as a key to understanding the mechanisms underlying both protein degradation and neurodegeneration. Here, we intend to summarize

\footnotetext{
* Correspondence: wgneuron@hotmail.com

${ }^{\dagger}$ Equal contributors

'Department of Neurology \& Neuroscience Institute, Ruijin Hospital affiliated to Shanghai Jiao Tong University School of Medicine, Shanghai 200025, China

Full list of author information is available at the end of the article
}

advances in the study of the endosomal-lysosomal system, with a focus on compartmentalized organization of trafficking routes, sorting machinery and their relationships to neurodegeneration.

\section{The endosomal-lysosomal system: a dynamic, interconnected vesicular network}

Membrane dynamics which affect protein degradation and recycling within cells plays a critical role in maintaining homeostasis. Macromolecules and transmembrane proteins at the plasma membrane destined for degradation could enter the endosomal-lysosomal system via three broadly defined routes: endocytosis, autophagy or phagocytosis $[4,5]$. Here we focus on the organization and functions of endocytic trafficking routes of protein/glycoprotein, and protein-bound lipid cargoes, preferentially over autophagy or phagocytosis.

The endocytic pathway is composed of a series of highly dynamic membrane-enclosed tubulo-vesicular 
structures. According to their different functions and roles in this system, several distinct types of compartments have been identified: early endosomes, recycling endosomes and late endosomes [6]. Additionally, lysosomes serve as organelles for storage of hydrolases, and are typically considered as a final destination where proteolytic degradation takes place. The membrane bilayer of endosomes and lysosomes creates an enclosed environment allowing an acidic $\mathrm{pH}$, which is optimal for many hydrolases and other enzymes. Compared to a cytoplasmic $\mathrm{pH}$ (of about 7.0), the endosomal and lysosomal lumen $\mathrm{pH}$ is maintained in a range of 6.5 to 4.5 , due to the activity of the ATP-dependent proton pumps present in the membrane of both endosomes and lysosomes [7]. Molecular trafficking and sorting along the endocytic pathway is regulated by the Rab family of small GTPases, which are critically important membrane association proteins. The Rabs function as molecular switches that alternate between two conformational states: the activated GTP-bound form and the GDPbound inactivated form [8]. Different Rab proteins have corresponding host organelles; therefore, they are often regarded as markers of different endosomal compartments.

Endosomes are dynamic, specialized compartments and undergo morphological and biological changes accompanied by vesicle trafficking. The maturation model states that endosomes along the endocytic pathway are transient and distinct compartments and, endosomes go through defined stages as they mature [6]. In this model, the process of endosomal maturation is characterized by four changes (Fig. 1): (1) an increasing number of intraluminal vesicles; (2) an increase in luminal acidification; (3) movement in space from the cell periphery towards the microtubule organizing center (MTOC); and (4) the switching of Rab proteins.

Initially, endocytosis (protein internalization) often starts at clathrin-coated pits that bud into vesicles derived from the plasma membrane. Within seconds after their formation, these vesicles can fuse with each other or with pre-existing early endosomes under the direction of the small GTPase Rab5 [9]. Early endosomes are generally formed in the peripheral cytoplasm with a slightly acidic intraluminal $\mathrm{pH}$ so that receptor cargo (ligands) can readily dissociate. The early endosome acts as a major sorting station, permitting the newly vacated receptors to recycle back to the cell surface for reuse, and directing dissociated ligands to be transported to late endosomes and finally to lysosomes for degradation. As mentioned, some membrane receptors along with membrane-bound lipids are transferred to recycling endosomes, returning to the plasma membrane, and this process is regulated by Rab4 and Rab11 [10, 11].
As internal vesicles bud inward from the membrane of the endosome, the number of intralumenal vesicles increases and this is an important transition in the maturation of early endosomes into late endosomes, and is the process whereby multivescular bodies (MVB) are created. The maturation from early to late endosomes is accompanied by the transition from association with Rab5 to Rab7, which is also known as "Rab conversion" [12]. Rab7 is a critical regulatory component that regulates transformation from early-to-late endosomes. In this process, endosomes move along a microtubule to the perinuclear region, regulated by interactions with dynein and kinesin and this process is accompanied by increasing intravacuolar acidification. The central role of late endosomes involves (1) the biogenesis of intralumenal vesicles and (2) they serve a role as a sink for sorting of ubiquitinated membrane proteins for eventual lysosomal degradation, both of which are executed by the ESCRT system of ubiquitin interacting and editing complexes [13]. At last, late endosomes or MVBs fuse with lysosomes and cargo proteins and intraluminal vesicles are degraded. It has been shown that late endosomes can be major sites of protein hydrolase activity acting on soluble proteins like ovalbumin taken up via earlier endocytosis, surpassing the activity found in lysosomes for these substrates, and so the entire endosomal-lysosomal compartment can be considered as a reservoir for hydrolases either active or inactive [14, 15]. Moreover, along the endosomal-lysosomal pathway, the concentration of acid hydrolases increases, while lumenal pH decreases, although activity varies by enzyme and not all are most active against all substrates in the lysosome [14]. In most neuronal cells, the $\mathrm{pH}$ of the lysosomal lumen can be as low as 4.5 [16]. Within this lumen, there are more than 50 hydrolases concentrated, and some of these show little to no sequence homology, suggesting convergent evolution. The fusion of late endosomes with lysosomes generally initiates the activation of hydrolase precursors or proenzymes, with a requirement for intraluminal $\mathrm{Ca}^{2+}$ and calmodulin [17], and prerequisite glycosylation with mannose-6-phosphate, which targets the hydrolases to receptors that concentrate them in the endosomal-lysosomal system [14]. Accordingly, lysosomes serve as the major site of activation of many proteolytic activities, even if their activity can be detected in other aspects of the endomembrane system.

The various stages of endocytosis with their branching decision tree for the sorting of receptors, receptor cargo, other transmembrane proteins, and hydrolase enzymes have a fundamental role in both membrane and protein processing, especially in neurons that require specialized regulation of endocytic and secretory pathways to ensure their normal function, particularly at synapses [18]. 


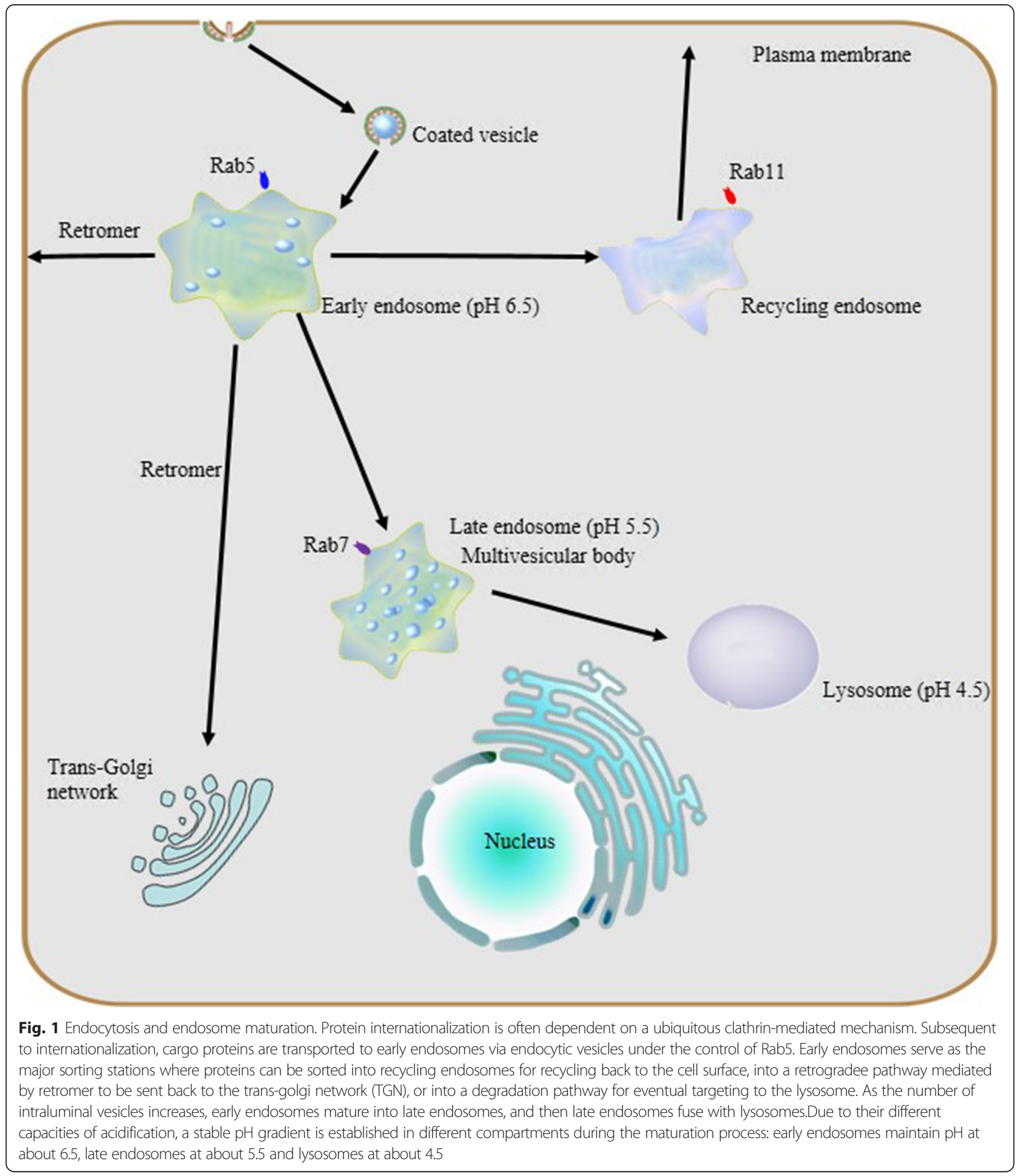

\section{A pH gradient established by vacuolar ATPase and chloride channels \\ Vacuolar ATP-dependent acidification}

As mentioned above, the endosomal and lysosomal compartments share at least one similar significant characteristic: low intraluminal $\mathrm{pH}$. These sealed acidic compartments provide an appropriate environment for optimal degradation of substrate cargo and recycling of their cognate receptors. As macromolecules are transported along the endosomal-lysosomal pathway, the internal $\mathrm{pH}$ of both endosomes and lysosomes decreases rapidly due to vacuolar acidification. Previous research 
revealed that the same $\mathrm{H}^{+}$-ATPase, also known as vacuolar ATPase (V-ATPase), acidifies both endosomes and lysosomes. V-ATPase, differs from $\mathrm{Na}^{+}, \mathrm{K}^{+}$-ATPase in the plasma membrane, $\mathrm{Ca}^{2+}$-ATPase in the sarcoplasmic reticulum, and $\mathrm{F}_{1}, \mathrm{~F}_{0}$-ATP synthase in mitochondria, in that it does not require a coupled influx of permeant anions [19]. Sulfhydryl alkylating reagents such as Nethylmaleimide inhibit the V-ATPase dependent acidification of the endosomal-lysosomal system [19-21] as well as the specific inhibitor Bafilomycin A1 [22, 23]. V-ATPase is a unique class of ATPase present throughout the membranes which constrain the endocytic pathway, including the trans golgi network (TGN). V-ATPase, as a protein complex, is composed of two multimeric subunits, $\mathrm{V}_{1}$ in the cytoplasmic domain and $V_{0}$ within the vacuolar membrane; the activity of V-ATPase depends on the dynamic assembly of these. V-ATPase is widely expressed in eukaryotic cells and serves as the master regulator of vesicular acidification in many subcellular membrane bound organelles. It also has important roles to play in vesicular trafficking and proteostasis.

\section{Chloride channel compensation}

Since V-ATPase is electrogenic, continuous proton influx across membranes would result in an everincreasing positive charge buildup in the lumen of endosomes and lysosomes. Since this potential difference would constitute an energetic barrier to maintenance of acidification, it is apparent that vacuoles must have a means of compensating. A series of studies have identified that chloride channels (CLC) conduct passive $\mathrm{Cl}^{-}$ion current, which compensates for proton accumulation by V-ATPase [24, 25]. In mammals, CLC proteins form a large family of nine members and among them, CLC3 to CLC7 localize to membranes of the endosomal-lysosomal system. These vesicular CLCs are thought to stimulate VATPase activity in endosomal-lysosomal compartments, thereby facilitating acidification. To do this, CLCs permit $\mathrm{H}^{+}$influx against a concentration gradient, but compensate by minimizing the electrochemical gradient, resulting in net regulation of the $\mathrm{pH}$ of vesicular compartments. In turn, CLC ion currents are regulated by the state of the intraluminal environment: for example, low lumenal $\mathrm{pH}$ is able to open CLC channel gating and $\mathrm{Cl}^{-}$currents. Even though there are still controversies over the importance of CLCs, a number of studies have provided evidence that CLC defects in the nervous system of mice led to endosomal $\mathrm{pH}$ elevation, and dysfunctional cellular protein degradation, causing phenotypes very similar to human neurodegenerative diseases, like $\mathrm{AD}$ and $\mathrm{PD}[26,27]$.

\section{Functions mediated by acidification}

An acidic lumenal environment is necessary for proper function as follows [14]: (1) internalized receptors need acidic conditions in order to release their ligands and recycle back to the membrane; (2) the $\mathrm{pH}$ gradient along the endosomal-lysosomal pathway might direct the movement and maturation of these organelles [15]; (3) many hydrolases have optimal function at acidic $\mathrm{pH}$; (4) acidic $\mathrm{pH}$ conditions may produce an environment favorable to oxidation reactions within the endosomal-lysosomal system.

\section{Two opposing cargo sorting systems: the retromer and ESCRT}

On entering the endosomal-lysosomal network, internalized protein cargo has two potential destinies: either it is trafficked and delivered to the late endosomes and terminally, to lysosomes for degradation (via the ESCRT system); or, it is sorted and transported to the TGN or the plasma membrane for reuse (via the retromer).

\section{ESCRT: sorting ubiquitinated proteins for degradation}

In the pathway promoting degradation, cargo proteins are transported into the intralumenal vesicles of endosomes for subsequent degradation. This process is initiated by a cargo-sorting receptor, namely ESCRT, such that target proteins are first captured in a signaldependent manner. At the endosomal membrane, it is now evident that the ESCRT complexes serve to recognize and sort ubiquitinated endosomal proteins for degradation [13]. The machinery and molecular basis of this ubiquitin-dependent endosomal sorting is emerging through many studies in yeast as well as mammalian cells, some of which we highlight below.

The ESCRT machinery consists of four distinct protein complexes: ESCRT-0, I, II, and III, named for their order of recruitment and function in triggering ubiquitindependent sorting of cargo proteins into the MVBs of endosomes [28]. Advances in biochemistry and cell biology have provided a good picture of the architecture and interactions of the ESCRTs. The "conveyor belt model" for ESCRT complex function proposes that these complexes function sequentially to sort ubiquitinated proteins into MVBs, starting with ESCRT-0 and ending with ESCRT-III.

ESCRT-0, the least evolutionarily conserved of the ESCRT complexes, contains multiple ubiquitin-binding subunits or activities and is able to retain ubiquitylated proteins in or at the endosomal membrane, but its affinity for ubiquitin is very low. It is, however, able to support the clustering of ubiquitinated membrane receptors into patches of membrane, thereby increasing the local concentration of such receptors. Once ESCRT-0 has 
bound to an ubiquitinated protein, ESCRT-I, which has higher affinity, can take on the role of binding to the ubiquitinated substrate protein. ESCRT-I as well as ESCRT-II each have just one ubiquitin-binding domain, so that it is hypothesized they require ESCRT-0 for concentration of ubiquitin-tagged cargo prior to their binding [13]. ESCRT-III comprises several small, highly charged subunits, assembling in a highly ordered manner. The ESCRT-III complex has moonlighting roles in cytokinesis and viral budding, but is known to harbor deubiquitinase activity that acts to release ubiquitin from substrates successfully moving down the ESCRT conveyor. ESCRT-III also has functions in the genesis of MVBs by supporting invagination and pinching off of the endosomal membrane in regions that have sequestered cargo $[29,30]$.

\section{Retromer: a singular path for retrograde retrieval}

Over the past couple of decades, there has been an increasing body of evidence implicating an ancient, evolutionarily conserved eukaryotic complex, which has come to be termed the "retromer." The primary function of the retromer is to conduct multiple cargo-sorting events, mediating retrograde retrieval from endosomes to the TGN [31]. In yeast, the retromer is comprised of five subunits, all encoded by vacuolar protein sorting (VPS) genes: Vps35-Vps29-Vps26 are a trimeric core, while Vps5-Vps17 is a dimer of sorting nexin (SNX) proteins. Even though SNX proteins differ significantly between species, the trimeric protein is highly conserved, which is also named the cargo-selective complex (CSC) for its role in recognizing cargo proteins [32]. In this recycling pathway, the principal cargoes of retrieval are unliganded receptors that may have been missorted to lysosomes.

Even though there remain significant gaps in our understanding of specific mechanisms of retrograde trafficking, it is clear that, as pioneering studies have indicated, the retromer has cargo-specific recognition capacity, and that there are different forms of the complex acting on distinct sets of cargo through CSC association with different sorting nexins [33]. It is notable that the CSC trimer, despite its role as the core functional component of the retromer complex, has no membranebinding activity and relies on nexins or Rab7 to execute its functions directed at membrane bound cargo. Previously, Vps35 was thought to provide the sole interface for cargo recognition, but recent studies have shown that other proteins such as Vps26, SNX3, and SNX27, are also implicated in cargo recognition in mammalian cells [31]. The sorting nexins recruit the CSC to endosomes and mediate its binding to the endosomal membrane. For example, SNX3, specifically recognizing phosphatidylinositol-3-monophosphate (which is enriched in early endosomes), can target the CSC to this compartment.

\section{Abnormalities and dysregulation of the endosomal-lysosomal system in neurodegeneration}

Increasing attention being paid to the endosomallysosomal system has begun to elucidate a relationship between endosomal-lysosomal defects and neurodegeneration. In particular, robust pathology implicating endosomal-lysosomal disruption in $\mathrm{AD}$ has been well characterized. Here, we focus preferably on $\mathrm{AD}$ as a example of neurodegenerative disease and we believe that AD represents a general model of neurodegenerative diseases on abnormalities of the endosomal-lysosomal system occur along a continuum that includes early endosome changes, dysregulated acidification and sorting component defects (Fig. 2).

\section{"APP, in the early endosome, with beta secretase": accomplices and bystanders}

Even though many details remain to be resolved, a striking body of evidence indicates that disturbed vesicular trafficking has special relevance in $\mathrm{AD}$ and other neurodegenerative diseases [3]. In particular, as just described, early endosomes mark the location of initial amyloidogenic processing of APP by beta-site APP cleaving enzyme 1 (BACE1) and dissociation of ApoE from its receptor, LDLR1, upon internalization and the extent to which these events occur is dependent upon the time of residence in this compartment, thus attracting much attention. ApoE and its receptor genetically and functionally interact with APP, to influence its endocytosis and degradation $[34,35]$, thereby modulating the severity of amyloid pathogenesis.

Studies have demonstrated that BACE1 and APP proteolytic fragments, $A ß$ and carboxyl-terminal APP fragment by BACE1 (ßCTF) are abundant in early endosomes [1, 36, 37]. For example, in cell models of $\mathrm{AD}$ pathology, the $\mathrm{BCTF}$ has been found to be accumulated in early endosomes and it can disrupt neuronal ionic homeostasis in a concentraction-dependent manner [38]. Meanwhile, the dysfuction of BACE1 retrograding from the endosomes into the TGN, appears to enhance the production of $B C T F$, leading to an increase of $A ß$ in early endosomes. In the brains of $A D$ patients, Cataldo et al. found that, even at earliest stage of disease when neurons exhibit enlarged Rab5positive endosomes containning $A ß$ immnoreactivity [39]. Moreover, Aß1-42 may also accumulate in lysosomes, disrupting the lysosomal membrane [40]. As a consequence, it has been noted that ectopic, elevated levels of lysosomal hydrolases can be observed in the CSF of individuals with AD [41]. It was first reported 


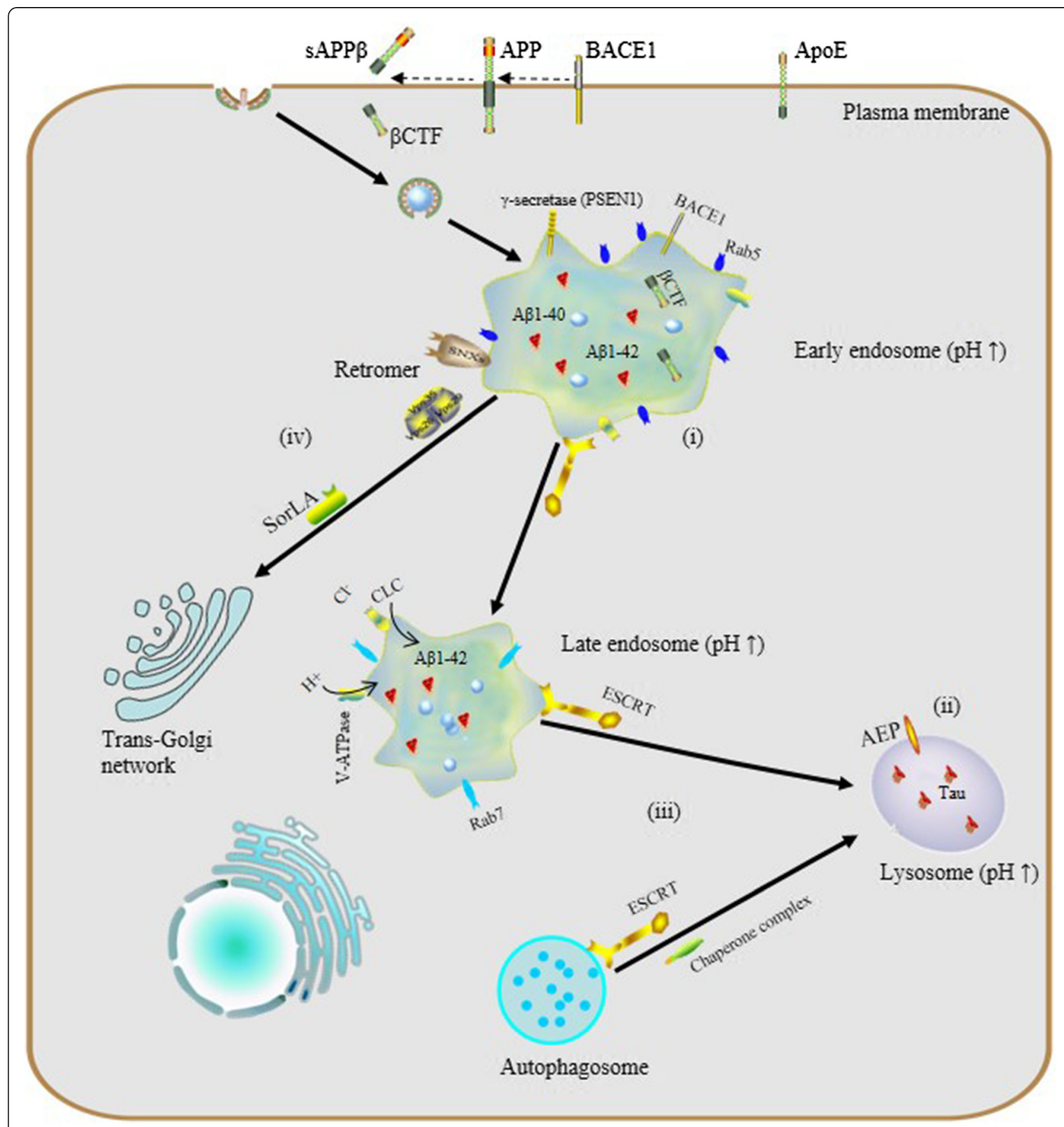

Fig. 2 The endosomal-lysosomal system and AD. (i) endosomal enlargement, Rab5 overexpression and Aß accumulation; (ii) dysregulated endosomal-Iysosomal acidification, pH elevation and tau aggregation; (iii) dysfunctional ESCRT complexes, defective autophagy and accumulation of intraluminal ubiquitinated proteins; (iv) defects of retromer: reduction of Vps26, Vps35 and SorLA disrupts the trafficking and processing of APP

almost two decades ago that in familial AD, Rab5 is overexpressed or stabilized post-translationally concommitant with a very marked increase in pyramidal neuron early endosome volume, and this results in increased processing of APP, a sorting imbalance, and decreases in neurotrophin receptor expression [42, 43]. Enlargement of the early endosome compartment has been considered as the earliest pathological change in $\mathrm{AD}$, even occurring decades before clinical symptoms are evident [44]. Further, swollen endosomal profiles have been postulated to represent activation of the endosomallysosomal pathway, involving increased rates of endocytosis and vesicular turnover. Consistent with this, studies using Rab5 as a specific endosomal marker have identified 
a relationship between increased endocytosis and an enlargement in endosome size and volume [45]. Interestingly, a number of Rabs that regulate the flux of traffic through early endosomes directly or in adjacent compartments have been found by RNAi screen to be either positive or negative regulators of $A ß$ production in heterologous mutant APP expressing cell lines [46]. Another recent review that delves into effects of interactors in regulating traffic of APP and secretases, particularly considering events increasing their co-location throughout the endomembrane system as opportunities for alternative APP processing events [47].

Even though it is established that $\mathrm{AD}$ is a heterogeneous disease, perturbation of the neuronal endosomallysosomal pathway is one cellular feature shared in common by all subtypes of $\mathrm{AD}$. Therefore, it is tempting to speculate that cellular pathological changes affecting this system are early and essential initiating events in $\mathrm{AD}$ pathogenesis, regardless of disease subtype and genetic predisposition.

\section{Dysregulated acidification, cellular indigestion?}

Endosomal-lysosomal $\mathrm{pH}$ defects are an emerging theme in mechanisms underlying a number of neurodegenerative diseases. To date, results from experiments in vivo and in vitro have revealed the importance of proper vesicular $\mathrm{pH}$ balance and optimal acidification in transporting and degrading cargo via the endocytic pathway $[48,49]$. For instance, Lee et al. reported that, in presenilin1 (PSEN1)-deleted blastocysts, defective lysosome acidification was observed with a substaintially elevated lysosomal PH of 5.4 and PSEN1 is essential for the transport of mature V0a1 subunites of V-ATP to lysosomes for their acidification and proteolysis [50]. Specifically, dysregulation of acidification and intracellular $\mathrm{pH}$ perturbation could influence the activity of enzymes in endomembrane compartments, resulting in impaired clearance of protein aggregates downstream of elevated endomembrane system $\mathrm{pH}$, or conversely, due to decreased cytoplasmic $\mathrm{pH}$. Regarding the latter, asparaginyl endopeptidase (AEP) is a typical $\mathrm{pH}$-sensitive protein hydrolase the activity of which depends on the acidic $\mathrm{pH}$ of vesicular compartments. Predominantly localized in late endosomes, asparaginyl endopeptidase (AEP) specifically cleaves substrates with an asparagine residue at the P1 site. It is known that AEP can undergo reversible $\mathrm{pH}$-dependent autoproteolytic activation, and in normal conditions, full-length pro-AEP is inactive [51]. As $\mathrm{pH}$ decreases from neutral to acidic, the activity of AEP gradually increases, such that it is partially activated at $\mathrm{pH} 4.5$ and fully activated at $\mathrm{pH} 3.5$, via removal of a cap that covers the active site. In AD patients, lysosomal acidification may be defective and it has been shown that the intracellular $\mathrm{pH}$ of neurons gradually decreases with aging [52] and more so with lactic acid elevation seen in AD cortex [53], so ectopic AEP activation or activity after leakage of active enzyme from late endosomes or lysosomes may be increased. AEP is involved in pathological tau degradation. Specifically, AEP generates tau fragments that form insoluble fibrils and result in neurotoxicity and neuropathological changes in $\mathrm{AD}[54,55]$.

Increased endosome and lysosome $\mathrm{pH}$ is expected to have global effects on the proteome, particularly membrane proteins which rely on this pathway for their regulation and degradation. Interestingly, studies of microglia in culture have shown that in the absence of inflammatory IL-6 signaling, microglia do not achieve a sufficiently low lysosomal $\mathrm{pH}$ to degrade $\mathrm{A} ß$, while after stimulation, CLC7 trafficking to lysosomes increases and $\mathrm{pH}$ drops sufficiently to improve $\mathrm{A} ß$ clearance $[49,56]$. We have recently performed a systematic look at the proteomic effects of defective endosomal-lysosomal $\mathrm{pH}$ in a cellular model, in order to develop a better understanding of the global changes in the proteome that follow inhibition of V-ATPase and could be considered together as a signature or biomarker of defective vacuolar acidification $[57,58]$, which would be expected to have an overlap with changes seen in $\mathrm{AD}$ and/or other conditions which may be subject to this often agedependent defect. Indeed, blocking lysosomal degradation with bafilomycin A1 affects a significant increase in global K63 polyubiquitin linkages, which also occurs in $\mathrm{AD}$, but $\mathrm{AD}$ brain global ubiquitin linkage profiling shows changes in other linkages as well [59]. Since K63 linked ubiquitin is not targeted to the proteasome, but does increase with V-ATPase acidification in the model of lysosomal insufficiency, the increase in K63 linkages seen in $\mathrm{AD}$ implicates accumulation of ubiquitinated proteins with obligate ESCRT-mediated degradation. Thus, trafficking, inflammatory signaling, and cell-type specific roles of dynamic lysosomal acidification are becoming increasingly appreciated for potential roles in AD pathogenesis.

\section{Roles of sorting components in $A D$ The ESCRTs}

A number of neurodegenerative diseases, including $\mathrm{AD}$, are characterized by accumulation of intracellular ubiquitinated proteins which can be actively collected into aggregates that are usually targeted to autophagosomes, thus implicating defective autophagy. The ESCRT complexes are involved in multiple cellular processes, including efficient fusion of autophagic vesicles for bulk degradation of cargo proteins. Autophagy serves as an intracellular clearance mechanism, preventing the accumulation of proteins that disrupt neuronal function and eventually lead to neurodegeneration $[60,61]$. 
Interestingly, in early stages of $\mathrm{AD}$, autophagy in neurons is activated, but becomes compromised as the disease progresses. There are more and more studies to identify the signal molecular machinery involving autophagy and the ESCRTs. Studies indicate that the ESCRT machinery is not only involved in sorting ubiquitinated cargo but also in initiating vesicle formation within endosomes becoming MVBs [62]. The most direct evidence for an ESCRT role in protection of neurons from protein accumulation and neurodegeneration has been seen upon overexpression of charged multivesicular body protein $2 \mathrm{~B}$ (CHMP2B), which is a subunit of the ESCRT-III complex. CHMP2B overexpression in neurons impairs lysosomal degradation of internalized proteins, resulting in accumulation of autophagosome, dendritic retraction and neuronal loss [63]. Typically, the autophagic pathway converges with the endocytic pathway at a point where mature autophagosomes fuse with MVBs. Compromised ESCRT function blocks the maturation and the proper turnover of autophagosomes, while functional ESCRT complexes are required for autophagic fusion and efficient degradation $[64,65]$.

\section{The retromer}

Over the years, researchers have studied neurodegenerative diseases arising from defects of retromer sorting proteins [66-68]. Particular interest has been taken in the link between retromer function to $\mathrm{AD}$ pathogenesis [69]. First, it was proposed that the retromer mediates endosome-to-TGN traffic of beta secretase, particularly BACE1, a transmembrane protein and enzyme that cleaves APP, promoting amyloidogenic and neurotoxic release of the $B C T F$ that is the precursor of $A ß$ species, as well as the C-terminal fragment APP intracellular domain (AICD), and this event is considered to be upstream of neurotoxic effects resulting from BACE1 activity [70]. Dysfunction of the retromer increases the concentration of BACE1 in the endosomal compartments, thereby providing more of an opportunity for the cleavage of APP, Aß production and ultimately,extracellular $A ß$-containing plaques $[66,71]$. In the brains of patients with AD, expression of Vps26 and Vps35 is reduced. Since these are two critical components of the retromer CSC trimer that recognize retromer cargo including BACE1, loss of function for the CSC would be expected to promote amyloidogenesis. Indeed, another study found that Vps35 mutation leads to up-regulation of $A ß$ generation in mouse models [72]. Stabilizing the retromer complex via a drug-like chemical chaperone was found to decrease $A ß$ production and increase neuroprotective alpha cleavage, probably by shifting APP out of early endosomes and improving functional retromer complex levels [73].
Interestingly, the above mentioned chemical chaperone also shifted the localization of SorLA, which associates with both Vps26 and APP, modulating amyloidogenic cleavage of APP [74] by upregulating the sorting of APP into endosomal compartments [75]. Recently, a comprehensive study proved that reduced SorLA expression is associated with mild cognitive impairment $(\mathrm{MCI})$ and $\mathrm{AD}$ [76]. In cultured hippocampal neurons, studies have confirmed important roles of SorLA in trafficking and processing of APP. SorLA direct Aß peptides to late endosomes and lysosomes, consequently, promoting degradation and clearance [77, 78].

Finally, it is important to point out that the general processes of endocytosis and endosomal-lysosomal dysregulations above-mentioned, have profoundly distinct implications for potential functions associated with other neurodegenerative diseases, such as PD, ALS, and Frontotemporal lobe degeneration (FTLD).

\section{Conclusions and perspective}

The endosomal-lysosomal system is a complex and highly dynamic process, where internalized transmembrane proteins, receptors, receptor ligands, and some soluble extracellular proteins are transported, sorted, and/or degraded. In recent years, particular attention has been paid to the endosomal-lysosomal system because it is involved in almost all of the neurodegenerative diseases, even though how it does so in each still remains unclear. Ongoing future studies will investigate both common and cell-type (or even local membrane region) specific trafficking and proteostasis pathways involving the endosomal-lysosomal system as well as the larger endomembrane system. For example, a better understanding of distinct roles that ubiquitination plays in ESCRT-mediated proteostasis (and even lipid droplet homeostasis [79] which appears to be dysregulated in glia in neurodegeneration $[47,80]$ ) could help to predict and ultimately therapeutically address the onset and progression of neurodegenerative diseases for specific individuals or sub-populations. This milieu of membrane-bound proteins that dynamically sorts cargo enriched for signaling, inflammation, and neurotrophic functions-among others-promises to provide a mother lode of new therapeutic targets for amelioriating neurodegenerative diseases, but the exploration also promises to be challenging, requiring the development of novel techniques and insight.

\section{Competing interests}

The authors declare that they have no competing interests.

\section{Authors' contributions}

$\mathrm{YH}$ and ED wrote the manuscript. RR and GW critically revised the manuscript and provided insightful thoughts. All authors read and approved the final manuscript. 


\section{Acknowledgement}

This study was supported by Shanghai Pujiang Program (No. 15PJ1405400). We thank Dr. Lian Li at the Department of Pharmacology, Emory University School of Medicine, Atlanta, GA 30322 USA for her critical reading.

\section{Author details}

${ }^{1}$ Department of Neurology \& Neuroscience Institute, Ruijin Hospital affiliated to Shanghai Jiao Tong University School of Medicine, Shanghai 200025, China. ${ }^{2}$ Department of Biochemistry, Center for Neurodegenerative Diseases, Emory University School of Medicine, Atlanta, GA 30322, USA.

\section{Received: 6 August 2015 Accepted: 22 September 2015}

\section{Published online: 30 September 2015}

\section{References}

1. Tan J, Evin G. Beta-site APP-cleaving enzyme 1 trafficking and Alzheimer's disease pathogenesis. J Neurochem. 2012;120(6):869-80.

2. Wu J, Petralia RS, Kurushima H, Patel H, Jung MY, Volk L, et al. Arc/Arg3.1 regulates an endosomal pathway essential for activity-dependent betaamyloid generation. Cell. 2011;147(3):615-28.

3. Neefjes J, van der Kant R. Stuck in traffic: an emerging theme in diseases of the nervous system. Trends Neurosci. 2014;37(2):66-76.

4. Tooze SA, Abada A, Elazar Z. Endocytosis and autophagy: exploitation or cooperation? Cold Spring Harb Perspect Biol. 2014;6(5):a018358.

5. Lamb CA, Dooley HC, Tooze SA. Endocytosis and autophagy: Shared machinery for degradation. Bioessays. 2013;35(1):34-45.

6. Huotari J, Helenius A. Endosome maturation. EMBO J. 2011;30(17):3481-500.

7. Diering $\mathrm{GH}$, Numata M. Endosomal pH in neuronal signaling and synaptic transmission: role of $\mathrm{Na}+/ \mathrm{H}+$ exchanger NHE5. Frontiers in Physiology. $2014 ; 4: 412$.

8. Villarroel-Campos D, Gastaldi L, Conde C, Caceres A, Gonzalez-Billault C. Rab-mediated trafficking role in neurite formation. J Neurochem. 2014;129(2):240-8

9. Laifenfeld D, Patzek LJ, McPhie DL, Chen Y, Levites Y, Cataldo AM, et al. Rab5 mediates an amyloid precursor protein signaling pathway that leads to apoptosis. J Neurosci. 2007;27(27):7141-53.

10. Li X, DiFiglia M. The recycling endosome and its role in neurological disorders. Prog Neurobiol. 2012;97(2):127-41.

11. Hsu WW, Prekeris R. Transport at the recycling endosome. Curr Opin Cell Biol. 2010;22(4):528-34.

12. Poteryaev D, Datta S, Ackema K, Zerial M, Spang A. Identification of the switch in early-to-late endosome transition. Cell. 2010;141(3):497-508.

13. Raiborg C, Stenmark H. The ESCRT machinery in endosomal sorting of ubiquitylated membrane proteins. Nature. 2009;458(7237):445-52.

14. Pillay CS, Elliott E, Dennison C. Endolysosomal proteolysis and its regulation. Biochem J. 2002;363(Pt 3):417-29.

15. Tjelle TE, Brech A, Juvet LK, Griffiths G, Berg T. Isolation and characterization of early endosomes, late endosomes and terminal lysosomes: their role in protein degradation. J Cell Sci. 1996;109(Pt 12):2905-14.

16. Ishida Y, Nayak S, Mindell JA, Grabe M. A model of lysosomal pH regulation. J Gen Physiol. 2013;141(6):705-20.

17. Luzio JP, Gray SR, Bright NA. Endosome-lysosome fusion. Biochem Soc Trans. 2010;38(6):1413-6.

18. Wang D, Chan CC, Cherry S, Hiesinger PR. Membrane trafficking in neuronal maintenance and degeneration. Cell Mol Life Sci. 2013;70(16):2919-34.

19. Maxson ME, Grinstein S. The vacuolar-type H(+)-ATPase at a glance - more than a proton pump. J Cell Sci. 2014;127(23):4987-93.

20. Breton S, Brown D. Regulation of luminal acidification by the V-ATPase. Physiology (Bethesda). 2013;28(5):318-29.

21. Morel N, Poea-Guyon S. The membrane domain of vacuolar H(+)ATPase: a crucial player in neurotransmitter exocytotic release. Cell Mol Life Sci. 2015;72(13):2561-73.

22. Yuan N, Song L, Zhang S, Lin W, Cao Y, Xu F, et al. Bafilomycin A1 targets both autophagy and apoptosis pathways in pediatric B-cell acute lymphoblastic leukemia. Haematologica. 2015;100(3):345-56.

23. Wu YC, Wu WK, Li Y, Yu L, Li ZJ, Wong CC, et al. Inhibition of macroautophagy by bafilomycin A1 lowers proliferation and induces apoptosis in colon cancer cells. Biochem Biophys Res Commun. 2009;382(2):451-6.

24. Stauber T, Jentsch TJ. Sorting motifs of the endosomal/lysosomal CLC chloride transporters. J Biol Chem. 2010;285(45):34537-48.
25. Scheel O, Zdebik AA, Lourdel S, Jentsch TJ. Voltage-dependent electrogenic chloride/proton exchange by endosomal CLC proteins. Nature. 2005;436(7049):424-7.

26. Syntichaki P, Samara C, Tavernarakis $N$. The vacuolar $\mathrm{H}+-$ ATPase mediates intracellular acidification required for neurodegeneration in C. elegans. Curr Biol. 2005;15(13):1249-54

27. Jentsch TJ, Poet M, Fuhrmann JC, Zdebik AA. Physiological functions of $\mathrm{CLC} \mathrm{Cl-} \mathrm{channels} \mathrm{gleaned} \mathrm{from} \mathrm{human} \mathrm{genetic} \mathrm{disease} \mathrm{and} \mathrm{mouse}$ models. Annu Rev Physiol. 2005;67:779-807.

28. Stuffers S, Brech A, Stenmark H. ESCRT proteins in physiology and disease. Exp Cell Res. 2009;315(9):1619-26.

29. Hurley JH. ESCRT complexes and the biogenesis of multivesicular bodies. Curr Opin Cell Biol. 2008;20(1):4-11.

30. Teis D, Saksena S, Judson BL, Emr SD. ESCRT-II coordinates the assembly of ESCRT-III filaments for cargo sorting and multivesicular body vesicle formation. The EMBO Journal. 2010;29(5):871-83.

31. Burd C, Cullen PJ: Retromer: a master conductor of endosome sorting. Cold Spring Harb Perspect Biol 2014, 6(2). doi:10.1101/cshperspect.a016774.

32. Bonifacino JS, Hurley JH. Retromer. Curr Opin Cell Biol. 2008;20(4):427-36

33. McGough IJ, Cullen PJ. Recent Advances in Retromer Biology. Traffic 2011;12(8):963-71.

34. Ulery PG, Beers J, Mikhailenko I, Tanzi RE, Rebeck GW, Hyman BT, et al Modulation of beta-amyloid precursor protein processing by the low density lipoprotein receptor-related protein (LRP). Evidence that LRP contributes to the pathogenesis of Alzheimer's disease. J Biol Chem. 2000;275(10):7410-5.

35. Kang DE, Pietrzik CU, Baum L, Chevallier N, Merriam DE, Kounnas MZ, et al. Modulation of amyloid beta-protein clearance and Alzheimer's disease susceptibility by the LDL receptor-related protein pathway. J Clin Invest. 2000;106(9):1159-66.

36. Rajendran L, Schneider A, Schlechtingen G, Weidlich S, Ries J, Braxmeier T, et al. Efficient inhibition of the Alzheimer's disease beta-secretase by membrane targeting. Science. 2008;320(5875):520-3.

37. Vassar R, Bennett BD, Babu-Khan S, Kahn S, Mendiaz EA, Denis P, et al. Beta-secretase cleavage of Alzheimer's amyloid precursor protein by the transmembrane aspartic protease BACE. Science. 1999;286(5440):735-41.

38. Kim HS, Park CH, Cha SH, Lee JH, Lee S, Kim Y, et al. Carboxyl-terminal fragment of Alzheimer's APP destabilizes calcium homeostasis and renders neuronal cells vulnerable to excitotoxicity. FASEB J. 2000;14(11):1508-17.

39. Cataldo AM, Petanceska S, Terio NB, Peterhoff CM, Durham R, Mercken M, et al. Abeta localization in abnormal endosomes: association with earliest Abeta elevations in AD and Down syndrome. Neurobiol Aging. 2004;25(10):1263-72.

40. Ling D, Song HJ, Garza D, Neufeld TP, Salvaterra PM. Abeta42-induced neurodegeneration via an age-dependent autophagic-lysosomal injury in Drosophila. PLoS One. 2009;4(1):e4201.

41. Nixon RA. Endosome function and dysfunction in Alzheimer's disease and other neurodegenerative diseases. Neurobiol Aging. 2005;26(3):373-82

42. Cataldo AM, Barnett $J$, Pieroni C, Nixon RA. Increased neuronal endocytosis and protease delivery to early endosomes in sporadic Alzheimer's disease: neuropathologic evidence for a mechanism of increased betaamyloidogenesis. J Neurosci. 1997;17(16):6142-51.

43. Ginsberg SD, Alldred MJ, Counts SE, Cataldo AM, Neve RL, Jiang Y, et al. Microarray analysis of hippocampal CA1 neurons implicates early endosomal dysfunction during Alzheimer's disease progression. Biol Psychiatry. 2010;68(10):885-93.

44. Cataldo AM, Peterhoff CM, Troncoso JC, Gomez-Isla T, Hyman BT, Nixon RA. Endocytic pathway abnormalities precede amyloid beta deposition in sporadic Alzheimer's disease and Down syndrome: differential effects of APOE genotype and presenilin mutations. Am J Pathol. 2000;157(1):277-86

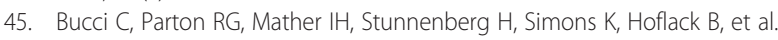
The small GTPase rab5 functions as a regulatory factor in the early endocytic pathway. Cell. 1992;70(5):715-28.

46. Udayar V, Buggia-Prévot V, Guerreiro Rita L, Siegel G, Rambabu N, Soohoo Amanda L, et al. A Paired RNAi and RabGAP Overexpression Screen Identifies Rab11 as a Regulator of $\beta$-Amyloid Production. Cell Reports. 2013;5(6):1536-51.

47. Jiang S, Li Y, Zhang X, Bu G, Xu H, Zhang YW. Trafficking regulation of proteins in Alzheimer's disease. Mol Neurodegener. 2014;9:6. 
48. Bonnet U, Scherbaum N, Wiemann M. The endogenous alkaloid harmane: acidifying and activity-reducing effects on hippocampal neurons in vitro. Prog Neuropsychopharmacol Biol Psychiatry. 2008;32(2):362-7.

49. Majumdar A, Capetillo-Zarate E, Cruz D, Gouras GK, Maxfield FR. Degradation of Alzheimer's amyloid fibrils by microglia requires delivery of ClC-7 to lysosomes. Mol Biol Cell. 2011;22(10):1664-76.

50. Lee JH, Yu WH, Kumar A, Lee S, Mohan PS, Peterhoff CM, et al. Lysosomal proteolysis and autophagy require presenilin 1 and are disrupted by Alzheimer-related PS1 mutations. Cell. 2010;141(7):1146-58.

51. Zhao L, Hua T, Crowley C, Ru H, Ni X, Shaw N, et al. Structural analysis of asparaginyl endopeptidase reveals the activation mechanism and a reversible intermediate maturation stage. Cell Res. 2014;24(3):344-58

52. Forester BP, Berlow YA, Harper DG, Jensen JE, Lange N, Froimowitz MP, et al. Age-related changes in brain energetics and phospholipid metabolism. NMR in Biomedicine. 2010;23(3):242-50.

53. Mastrogiacomo F, Bergeron C, Kish SJ. Brain a-Ketoglutarate Dehydrotenase Complex Activity in Alzheimer's Disease. J Neurochem. 1993;61(6):2007-14.

54. Zhang Z, Song M, Liu X, Kang SS, Kwon IS, Duong DM, et al. Cleavage of tau by asparagine endopeptidase mediates the neurofibrillary pathology in Alzheimer's disease. Nat Med. 2014;20(11):1254-62.

55. Wolfe DM, Lee JH, Kumar A, Lee S, Orenstein SJ, Nixon RA. Autophagy failure in Alzheimer's disease and the role of defective lysosomal acidification. Eur J Neurosci. 2013:37(12):1949-61.

56. Majumdar A, Cruz D, Asamoah N, Buxbaum A, Sohar I, Lobel P, et al. Activation of Microglia Acidifies Lysosomes and Leads to Degradation of Alzheimer Amyloid Fibrils. Mol Biol Cell. 2007;18(4):1490-6.

57. Dehay B, Martinez-Vicente M, Caldwell GA, Caldwell KA, Yue Z, Cookson MR, et al. Lysosomal impairment in Parkinson's disease. Mov Disord. 2013;28(6):725-32

58. Appelqvist $H$, Waster $P$, Kagedal K, Ollinger K. The lysosome: from waste bag to potential therapeutic target. J Mol Cell Biol. 2013;5(4):214-26.

59. Dammer EB, Na CH, Xu P, Seyfried NT, Duong DM, Cheng D, et al. Polyubiquitin Linkage Profiles in Three Models of Proteolytic Stress Suggest the Etiology of Alzheimer Disease. J Biol Chem. 2011;286(12):10457-65.

60. Lee JA, Liu L, Gao FB. Autophagy defects contribute to neurodegeneration induced by dysfunctional ESCRT-III. Autophagy. 2009;5(7):1070-2.

61. Wang G, Mao Z. Chaperone-mediated autophagy: roles in neurodegeneration. Transl Neurodegener. 2014:3:20.

62. Babst M. MVB vesicle formation: ESCRT-dependent, ESCRT-independent and everything in between. Curr Opin Cell Biol. 2011;23(4):452-7.

63. Lee JA, Beigneux A, Ahmad ST, Young SG, Gao FB. ESCRT-III dysfunction causes autophagosome accumulation and neurodegeneration. Curr Biol. 2007;17(18):1561-7.

64. Wilson CM, Magnaudeix A, Yardin C, Terro F. Autophagy dysfunction and its link to Alzheimer's disease and type II diabetes mellitus. CNS Neurol Disord Drug Targets. 2014;13(2):226-46.

65. Tung YT, Wang BJ, Hu MK, Hsu WM, Lee H, Huang WP, et al. Autophagy: a double-edged sword in Alzheimer's disease. J Biosci. 2012;37(1):157-65.

66. Muhammad A, Flores I, Zhang H, Yu R, Staniszewski A, Planel E, et al. Retromer deficiency observed in Alzheimer's disease causes hippocampal dysfunction, neurodegeneration, and Abeta accumulation. Proc Natl Acad Sci U S A. 2008;105(20):7327-32.

67. Tsika E, Glauser L, Moser R, Fiser A, Daniel G, Sheerin UM, et al. Parkinson's disease-linked mutations in VPS35 induce dopaminergic neurodegeneration. Hum Mol Genet. 2014:23(17):4621-38.

68. Damseh N, Danson CM, Al-Ashhab M, Abu-Libdeh B, Gallon M, Sharma K, et al. A defect in the retromer accessory protein, SNX27, manifests by infantile myoclonic epilepsy and neurodegeneration. Neurogenetics. 2015;16(3):215-21.

69. Reitz C. The role of the retromer complex in aging-related neurodegeneration: a molecular and genomic review. Mol Genet Genomics. 2014;290(2):41327.doi:10.1007/s00438-014-0939-9. Epub 2014 Oct 21. Review.

70. Jiang Y, Mullaney KA, Peterhoff CM, Che S, Schmidt SD, Boyer-Boiteau A, et al. Alzheimer's-related endosome dysfunction in Down syndrome is Abeta-independent but requires APP and is reversed by BACE-1 inhibition. Proc Natl Acad Sci U S A. 2010;107(4):1630-5.

71. Sullivan CP, Jay AG, Stack EC, Pakaluk M, Wadlinger E, Fine RE, et al. Retromer disruption promotes amyloidogenic APP processing. Neurobiol Dis. 2011;43(2):338-45.
72. Wen L, Tang FL, Hong Y, Luo SW, Wang CL, He W, et al. VPS35 haploinsufficiency increases Alzheimer's disease neuropathology. J Cell Biol. 2011;195(5):765-79

73. Mecozzi VJ, Berman DE, Simoes S, Vetanovetz C, Awal MR, Patel VM, et al. Pharmacological chaperones stabilize retromer to limit APP processing. Nat Chem Biol. 2014;10(6):443-9.

74. Fjorback AW, Seaman M, Gustafsen C, Mehmedbasic A, Gokool S, Wu C, et al. Retromer binds the FANSHY sorting motif in SorLA to regulate amyloid precursor protein sorting and processing. J Neurosci. 2012;32(4):1467-80.

75. Buggia-Prevot $V$, Thinakaran $G$. Sorting the role of SORLA in Alzheimer's disease. Sci Transl Med. 2014;6(223):223fs228.

76. Willnow TE, Carlo AS, Rohe M, Schmidt V. SORLA/SORL1, a neuronal sorting receptor implicated in Alzheimer's disease. Rev Neurosci. 2010;21(4):315-29.

77. Bhalla A, Vetanovetz CP, Morel E, Chamoun Z, Di Paolo G, Small SA. The location and trafficking routes of the neuronal retromer and its role in amyloid precursor protein transport. Neurobiol Dis. 2012;47(1):126-34.

78. Schmidt V, Baum K, Lao A, Rateitschak K, Schmitz Y, Teichmann A, et al. Quantitative modelling of amyloidogenic processing and its influence by SORLA in Alzheimer's disease. EMBO J. 2012;31(1):187-200.

79. Arrese EL, Saudale FZ, Soulages JL. Lipid Droplets as Signaling Platforms Linking Metabolic and Cellular Functions. Lipid Insights. 2014;7:7-16.

80. Liu L, Zhang K, Sandoval H, Yamamoto S, Jaiswal M, Sanz E, et al. Glial lipid droplets and ROS induced by mitochondrial defects promote neurodegeneration. Cell. 2015;160(1-2):177-90

\section{Submit your next manuscript to BioMed Central and take full advantage of:}

- Convenient online submission

- Thorough peer review

- No space constraints or color figure charges

- Immediate publication on acceptance

- Inclusion in PubMed, CAS, Scopus and Google Scholar

- Research which is freely available for redistribution 